\title{
Adult dermatomyositis associated with benign ovarian teratoma: A case report
}

\author{
XIAOMIN YU ${ }^{1,2}$, DONGYAN QIN ${ }^{1}$, DEHUA MA $^{1}$ and QIN YAO ${ }^{1}$ \\ ${ }^{1}$ Department of Obstetrics and Gynaecology, Affiliated Hospital of Qingdao University, Qingdao, Shandong 266003; \\ ${ }^{2}$ Department of Obstetrics and Gynaecology, The Eighth People's Hospital of Qingdao, Qingdao, Shandong 266100, P.R. China
}

Received December 31, 2014; Accepted January 28, 2016

DOI: $10.3892 / \mathrm{ol} .2016 .4251$

\begin{abstract}
A 27-year-old female patient presented with a 3-month history of bilateral orbital and facial edema accompanied by skin erythema and heliotrope rash. The left lesion was more critical than the right. Limb muscles were occasionally sore. On physical examination, scattered hemorrhagic rashes were observed on the patient's face and neck. Upon laboratory testing, creatine kinase (CK) was markedly elevated at 1,543.2 U/1, while CK-MB isoenzyme (27.4 U/1), $\alpha$-hydroxybutyric dehydrogenase (188.4 U/1) and aspartate aminotransferase (65.3 U/1) were marginally elevated. The patient was diagnosed with dermatomyositis due to the dermatological features and elevated CK. Cancer marker analysis revealed positivity for carbohydrate antigen $125(68.15 \mathrm{U} / \mathrm{ml})$. Magnetic resonance imaging revealed thickening of the soft tissue of the left eyelid; furthermore, concurrent long T1- and T2-weighted signals (fat saturation) were compatible with inflammatory infiltration. Non-enhanced computed tomography identified that the right accessory region (right ovary and fallopian tube) had a cystic mass with a significant fat component and thickening of the anterior uterine wall. Gynecological ultrasound findings indicated an ovarian teratoma $(8.7 \times 7.8 \times 9.3 \mathrm{~cm})$ and uterine myoma $(3.6 \times 3.1 \mathrm{~cm})$. The patient's dermatological symptoms and laboratory results were significantly relieved one week after surgical removal of the teratoma with the aid of hydrocortisone, methylprednisolone and methotrexate therapy.
\end{abstract}

Correspondence to: Professor Qin Yao or Miss. Xiaomin Yu, Department of Obstetrics and Gynaecology, Affiliated Hospital of Qingdao University, 16 Jiangsu Road, Qingdao, Shandong 266003, P.R. China

E-mail: dr_yaoqin@163.com

E-mail: yuxiaomin_brain@163.com

Key words: dermatomyositis, inflammatory myopathies, benign ovarian teratoma, paraneoplastic syndromes, immunosuppressive therapy

\section{Introduction}

Dermatomyositis is a type of idiopathic inflammatory myopathy (1). Bohan and Peter (1) proposed five major criteria for the diagnosis dermatomyositis and another idiopathic inflammatory myopathy, polymyositis, in 1975: Progressive symmetrical weakness, muscle-biopsy evidence, including muscle fiber swelling, absence of striations and muscle fibers revealing vacuolar degeneration, elevated enzymes in skeletal muscle serum, abnormal electromyogram and dermatological features. Bohan and Peter (1) also suggested five subsets of myositis: Polymyositis, dermatomyositis, dermatomyositis associated with neoplasia, child dermatomyositis associated with vasculitis and polymyositis or dermatomyositis with associated collagen-vascular disease. Subsequent to this, Saoud et al (2) recognized an additional subset: Amyopathic dermatomyositis. Furthermore, Callen (3) previously reported that dermatomyositis is associated with malignant tumors. Teratomas are a type of germ cell tumor comprising well-differentiated tissues and 3 germ cell layers: Ectoderm, mesoderm and endoderm. They are divided into mature (benign) and immature (malignant) teratomas, and dermatomyositis may be associated with malignant or benign tumors. The current study presents a case of dermatomyositis accompanied by a benign ovarian teratoma.

\section{Case report}

A 27-year-old female patient with a 3-month history of bilateral orbital and facial edema accompanied by skin erythema and rash accepted treatment at the Department of Rheumatology of the Affiliated Hospital of Qingdao University (Qingdao, China) and was admitted on December 13, 2012. The left lesion was more critical than the right. Limb muscles were occasionally sore, however, the patient reported no joint pain, proximal limb weakness, fever or hypersensitivity to light. A muscle biopsy did not demonstrate characteristic alterations of perifascicular atrophy and inflammation in the patient. The patient accepted hydrocortisone (20 mg, orally once a day) treatment in the Department of Dermatology and Ophthalmology (Affiliated Hospital of Qingdao University). Following receipt of this treatment regime the patient's symptoms were mildly relieved. However, once treatment was terminated two weeks later, the symptoms became worse than upon presentation. The patient 
reported no weight loss, decreased appetite or history of infections, but had undergone an appendectomy due to appendicitis 20 years previously and was allergic to levofloxacin. The patient was unmarried, had no children, had reached menarche at 13 years of age and reported a regular menstrual cycle with no dysmenorrhea. Furthermore, a family history revealed that the mother had hypertension, the father was healthy and there was no family history of genetic diseases.

Examination of the patient revealed a temperature of $36.6^{\circ} \mathrm{C}$ (normal range, $36.9-37.3^{\circ} \mathrm{C}$ ), a pulse of 80 beats $/ \mathrm{min}$ (normal range, $60-100$ beats/min), a respiratory rate of 20 breaths/min (normal range, 18-22 breaths/min) and blood pressure of $120 / 80 \mathrm{mmHg}$ (normal range, 90-130/60-85 mmHg). Scattered hemorrhagic rashes were observed on the patient's face and neck, a number of which had formed ulcerations. There was no lymphadenopathy. Edema was observed on the face and eyelid, accompanied by characteristic dermatological lesions (heliotrope rash). However, the ears, eyeballs, nose, throat and mouth were normal. Stethoscopy of both lungs revealed clear and smooth breathing. The patient's cardiac rate was regular, and sounded strong with no murmur. The abdomen was flat, soft and not painful. Upon examination of the limbs and spine, the strength and tension of the limb muscles were normal. There were no erythematous macules on elbows, knees or medial ankles. Urinary, reproductive system and nervous system examinations were normal. The patient did not have weak neck flexors or Gower maneuver.

Laboratory analysis revealed a white blood cell count of $10.75 \times 10^{9}$ cells/ 1 (71.40\% neutrophils, $18.90 \%$ lymphocyte, $9.30 \%$ mononuclear cells, $0.30 \%$ eosinophils and $0.10 \%$ basophils; normal range, $4.00-10.00 \times 10^{9}$ cells $\left./ 1\right)$, a red blood cell count of $4.28 \times 10^{12}$ cells/1 (normal range, 3,50-5.00x $10^{12}$ cells $/ 1$ ), a hemoglobin level of $130.00 \mathrm{~g} / 1$ (normal range, $110.00-150.00 \mathrm{~g} / \mathrm{l}$ ), a hematocrit level of $41.10 \%$ (normal range, $37.00-47.00 \%$ ) and a platelet count of $202.00 \times 10^{9} / 1$ (normal

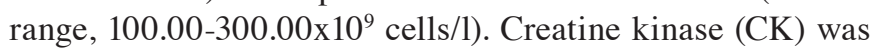
markedly elevated [1,543.2 U/1 (normal range, 0.0-170.0 U/1)], and CK-MB isoenzyme [27.4 U/1 (normal range, 0.0-17.0 U/1)], $\alpha$-hydroxybutyric dehydrogenase [188.4 U/1 (normal range, 72.0-182.0 U.1)] and aspartate aminotransferase [AST; $65.3 \mathrm{U} / 1$ (normal range, 0.0-42.0 U/1)] were mildly elevated. However, alanine aminotransferase (48.2 U/1; normal range, 0-60 U/1), lactate dehydrogenase (237.6 U/1; normal range, 91-245 U/1) and $\gamma$-glutamyl transferase levels $(9.5 \mathrm{U} / 1$; normal range, 0-64 U/1) were normal. Rheumatological evaluation revealed that the expression of the following anti-nuclear antibodies were all negative: Anti-SSA, anti-SSB, anti-Scl-70, anti-polymyositis/Scl, anti-Jo-1, anti-Sm and anti-double stranded DNA antibody. Cytoplasmic, perinuclear, protease 3 and myeloperoxidase anti-nuclear antibody, as well as anti-glomerular basement membrane antibody, were normal. The patient's immunoglobulin ( $\mathrm{Ig}$ ) levels ( $\mathrm{IgG}, 950 \mathrm{mg} / \mathrm{dl}$; normal range, 751-1560 mg/dl; IgA, $136 \mathrm{mg} / \mathrm{dl}$; normal range, 82-453 mg/dl; IgM, $232 \mathrm{mg} / \mathrm{dl}$; normal range, 46-304 mg/dl; IgE, $93.24 \mathrm{IU} / \mathrm{ml}$; normal range, $0-100 \mathrm{IU} / \mathrm{ml})$, complement levels $(\mathrm{C} 3,91.2 \mathrm{mg} / \mathrm{dl}$; normal range, 79-152 mg/dl; C4, $16.40 \mathrm{mg} / \mathrm{dl}$; normal range, $16-38 \mathrm{mg} / \mathrm{dl})$ and erythrocyte sedimentation rate $[10.5 \mathrm{~mm} / 1 \mathrm{~h}$ (normal range, 0.0-20.0 mm/1 h)] were also normal. Cancer marker analysis revealed positivity for carbohydrate antigen 125 [CA125; $68.15 \mathrm{U} / \mathrm{ml}$ (normal range, 0-35 U/ml)],
A

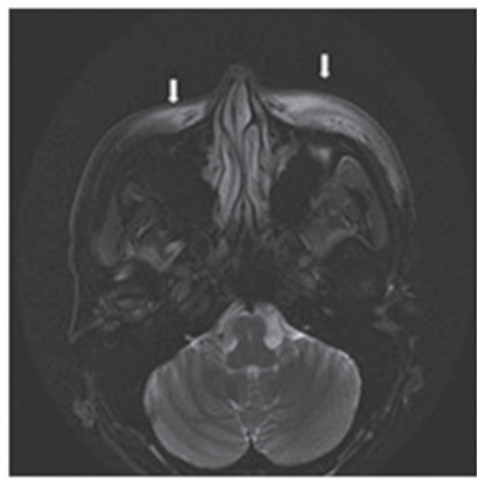

B

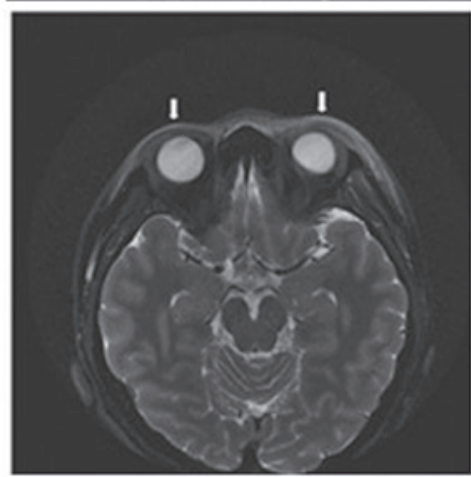

C

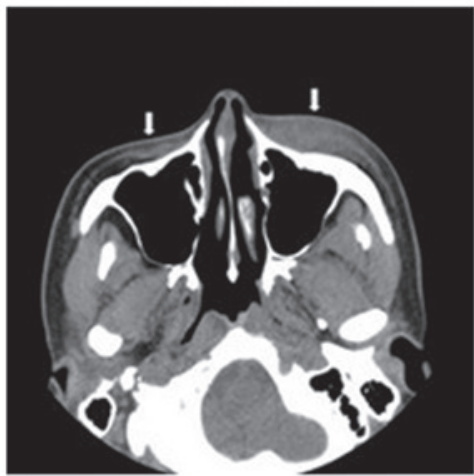

D

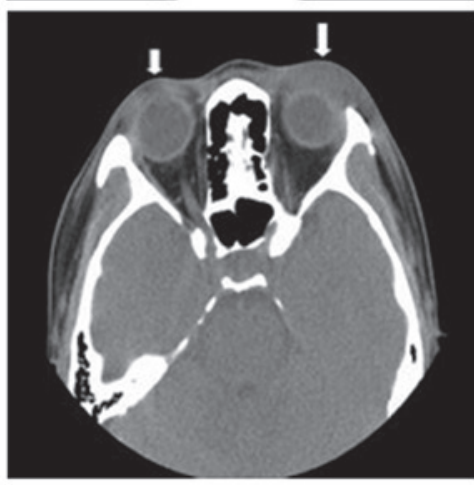

Figure 1. Magnetic resonance imaging (MRI) and computed tomography (CT) of the buccal region and peri-orbital inflammation. MRI revealed (A) bilateral buccal region and (B) bilateral eyelid thickening and swelling (indicated by white arrows), as well as subcutaneous long T2-weighted signal intensity. CT revealed (C) bilateral buccal region and (D) bilateral eyelid thickening with blurred subcutaneous fat clearance and high-density signals (indicated by white arrows).

and negativity for carcinoembryonic antigen, $\alpha$-fetoprotein, carbohydrate antigen 19-9, neuron-specific enolase, squamous cell carcinoma antigen and $\beta$-human chorionic gonadotropin.

Magnetic resonance imaging (MRI; Signa HDxt 3.0T; GEHealthcareLifeSciences, Chalfont,UK) revealed thickening 


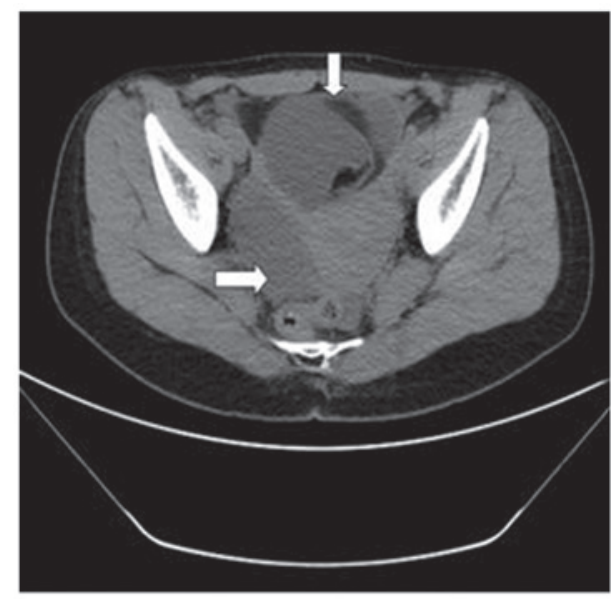

Figure 2. Computed tomography revealing a multilocular mass (indicated by white arrows) in the pelvic cavity. Density analysis indicated that the mass was partially composed of water and partially composed of fat.

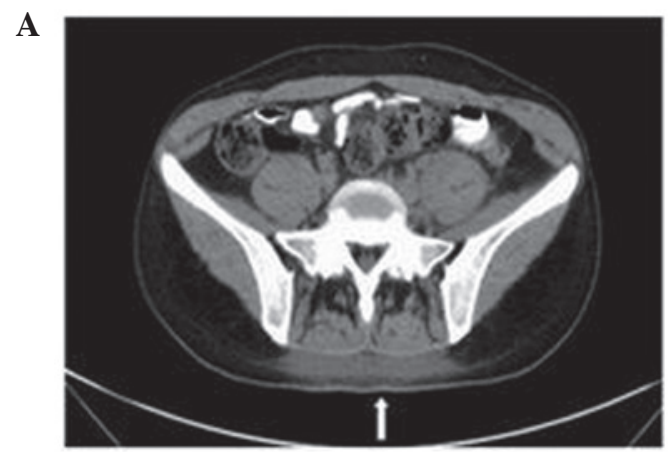

$\mathbf{B}$

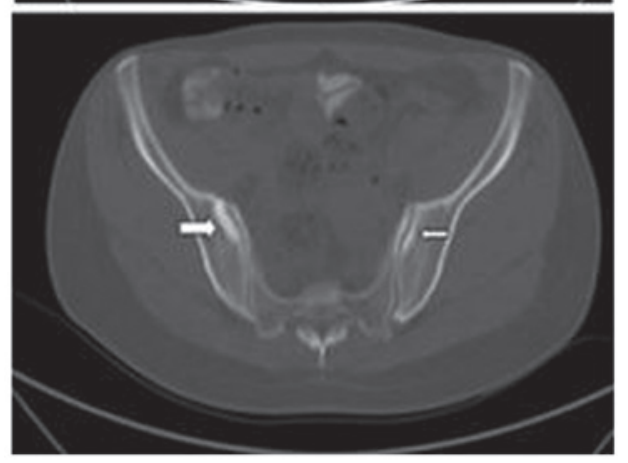

Figure 3. Computed tomography (CT) scan revealing (A) back inflammation (indicated by white arrow) and (B) sacroiliac arthritis (indicated by white arrows). These diagnoses were determined by observations of (A) blurred subcutaneous fat clearance of the back with high-density signals; and (B) bone destruction of the bilateral sacroiliac joint surface with high-density signals.

and swelling of the soft tissue of the bilateral eyelids and bilateral buccal regions; furthermore, subcutaneous long T1- and T2-weighted signal intensity (fat saturation) was compatible with inflammatory infiltration (Fig. 1A and B). There were no abnormal signals near the bilateral eyeballs or muscles around the eyes and posterior orbital. Computed tomography (CT; Brilliance iCT; Philips Healthcare, DA Best, The Netherlands) scans of the bilateral eyelids and bilateral buccal regions were consistent with the MRI findings (Fig. 1C and D). There were no obvious abnormalities upon pelvic X-ray and thoracic CT. B-mode ultrasound of the neck indicated no abnormalities in the thyroid or in lymph nodes located in neck subcutaneous tissue. Non-enhanced CT of the upper abdomen, lower abdomen and pelvic cavity revealed no significant abnormalities of the liver, kidney or bladder, respectively. However, a multilocular cystic mass in the pelvic cavity with a significant fat component was observed at the right accessory region, and the anterior wall of the uterus was thickened (Fig. 2). Additionally, CT indicated blurred subcutaneous fat clearance of the back and high-density signals (Fig 3A). Bone destruction and high-density signals were also observed on the bilateral sacroiliac joint surface (Fig. 3B). Gynecological ultrasound findings indicated an ovarian teratoma $(8.7 \times 7.8 \times 9.3 \mathrm{~cm})$ and uterine myoma $(3.6 \times 3.1 \mathrm{~cm})$.

Initially, the patient accepted treatment of hydrocortisone (20 mg, orally once a day) in the outpatient clinic of the Department of Dermatology and Ophthalmology (Affiliated Hospital of Qingdao University) for two weeks between November 2012 and December 2012. Following receipt of this treatment regime the patient's dermatological symptoms were mildly relieved. However, when treatment was terminated the dermatological symptoms worsened, and became more severe than at presentation. Subsequently, between December 2012 and January 2013 methylprednisolone (40 mg, intravenous drip; administered 4 times in the 1 month period) and methotrexate (5 mg, intravenous injection; administered 4 times in the 1 month period) therapy were administered at the Department of Rheumatology (Affiliated Hospital of Qingdao University). However, prior to surgery, there was no obvious relief in the patient's dermatological symptoms and the level of CK remained elevated (1131.8-1543.2 U/1). The patient was diagnosed with dermatomyositis due to the dermatological features, elevated $\mathrm{CK}$ and sacroiliac arthritis on December 25, 2012. The ovarian teratoma and uterine myoma were laprascopically removed in the Department of Obstetrics and Gynecology of the Affiliated Hospital of Qingdao University (Qingdao, China) on January 10, 2013, and diagnosed as benign right ovary cystic mature teratoma and uterine myoma by analysis of the biopsy. In order to perform this diagnosis, tissue was embedded in a paraffin block and sliced into $10-\mathrm{mm}$ sections using a microtome (SYD-S3020; Shenyang LongShou Electronic Instrument Co., Ltd., Shenyang, China), followed by hematoxylin and eosin staining (Shanghai Biyuntian Bio-Technology Co., Ltd., Shanghai, China). One week after surgical removal of the teratoma, the dermatological symptoms were significantly relieved. The level of CK was $248.7 \mathrm{U} / 1$, and the results of additional laboratory tests (CA125, CK-MB isoenzyme, AST) had returned to normal. The patient was discharged from hospital on January 21, 2013. Following discharge, the patient continued to receive oral methotrexate $(10 \mathrm{mg})$ and methylprednisolone $(28 \mathrm{mg})$. When the patient's $\mathrm{CK}$ was followed-up 2 weeks later, the level was normal (130 U/l) and dermatological symptoms were completely relieved. Follow-up consisted of observation of the facial skin lesions and the levels of $\mathrm{CK}, \mathrm{CKMB}$ and lactate dehydrogenase of the patient, which after two weeks of follow up were as follows: CK, $160 \mathrm{U} / 1$ (normal range, 0-170 U/1); CKMB, $15 \mathrm{U} / 1$ (normal range,0-17 U/1); lactate dehydrogenase, $230 \mathrm{U} / 1$ (normal range, 91-245 U/1). The patient is currently alive and well, with no signs of recurrence. 


\section{Discussion}

Callen and Wortmann (4) presented images of characteristic cutaneous lesions and clinical manifestation of dermatomyositis. In a study by Callen (2), it was reported that dermatomyositis is associated with malignant tumors, including those of the ovary, lung, pancreas, stomach, colon or rectum, as well as non-Hodgkin's lymphoma. Ibarra et al (5) described a case report of juvenile dermatomyositis accompanied by a benign teratoma. An 8-year-old female patient presented with of right arm pain, weakness in both legs and difficulty in arising from a seated or squatting position that was ongoing for 4 months, as well as 1 month of pain in the hips, ankles and knees (5). Following rheumatological evaluation, including elevated levels of $\operatorname{IgG}$ and $\mathrm{IgE}$, the patient was diagnosed with juvenile dermatomyositis. After surgical removal of the teratoma, the myositis, synovitis and cutaneous findings resolved over 4 months without additional therapy (5). Benign teratomas have also been associated with paraneoplastic syndromes, including paraneoplastic limbic encephalitis (6-10), opsoclonus-myoclonus syndrome (11), paraneoplastic polyarthritis (12) and autoimmune hemolytic anemia (13). Titulaer et al (14) performed a screen for tumors in paraneoplastic syndromes to aid the early detection of tumors.

In conclusion, to the best of our knowledge, the current case report provides a novel observation of dermatomyositis associated with a benign ovarian teratoma in an adult. The case also provides evidence that elevated levels of CK and the presence of heliotrope rash are associated with benign ovarian teratoma, despite the lack of progressive symmetrical weakness, muscle-biopsy evidence and abnormal electromyogram. We propose that benign ovarian teratoma may cause dermatomyositis (as in the present case it was proposed that the teratoma occurred before the dermatomyositis, as it was thought to be unlikely that the teratoma could have grown to a size of $8.7 \times 7.8 \times 9.3 \mathrm{~cm}$ in 3 months) in addition to paraneoplastic syndromes, as previously reported. The present study appears to expand the range of disease entities known to be caused by benign ovarian teratoma beyond paraneoplastic syndromes. The present study appears to expand the range of disease entities known to be caused by benign ovarian teratoma beyond the previously reported paraneoplastic syndromes. The current case also serves as a reminder of the process of dermatomyositis clinical diagnosis and treatment.

\section{References}

1. Bohan A and Peter JB: Polymyositis and dermatomyositis (first of two parts). N Engl J Med 292: 344-347, 1975.

2. Saoud B, Allali F and Hassouni NH: Amyopathic dermatomyositis. Joint Bone Spine 73: 318-320, 2006.

3. Callen JP: Relation between dermatomyositis and polymyositis and cancer. Lancet 357: 85-86, 2001.

4. Callen JP and Wortmann RL: Dermatomyositis. Clin Dermatol 24: 363-373, 2006.

5. Ibarra M,Chou P and Pachman LM: Ovarian teratoma mimicking features of juvenile dermatomyositis in a child. Pediatrics 128: e1293-e1296, 2011.

6. Sadalage G, Karim A and Jacob S: Autoimmune encephalitis screen-a review of rapid diagnostic screening in 600 patients over 5 years. J Neurol Neurosurg Psychiatry 84: e2, 2013.

7. Lee KG: Paraneoplastic limbic encephalitis associated with ovarian teratoma. Clin Med 12: 95-96, 2012.

8. Yang YW, Tsai CH, Chang FC, Lu MK and Chiu PY: Reversible paraneoplastic limbic encephalitis caused by a benign ovarian teratoma: Report of a case and review of literatures. J Neurooncol 80: 309-312, 2006.

9. Tanyi JL, Marsh EB, Dalmau J and Chu CS: Reversible paraneoplastic encephalitis in three patients with ovarian neoplasms. Acta Obstet Gynecol Scand 91: 630-634, 2012.

10. Hsu MH, Huang CC, Hung PL, Huang HM, Huang LT, Huang CC, Sheen JM, Huang SC and Chang YC: Paraneoplastic neurological disorders in children with benign ovarian tumors. Brain Dev 36: 248-253, 2014.

11. Fitzpatrick AS, Gray OM, McConville J and McDonnell GV: Opsoclonus-myoclonus syndrome associated with benign ovarian teratoma. Neurology 70: 1292-1293, 2008.

12. Wiese W, Alansari H, Tranchilda P and Madrid FF: Paraneoplastic polyarthritis in an ovarian teratoma. J Rheumatol 31: 1854-1857, 2004.

13. Kim I, Lee JY, Kwon JH, Jung JY, Song HH, Park YI, Ro E and Choi KC: A case of autoimmune hemolytic anemia associated with an ovarian teratoma. J Korean Med Sc 21: 365-367, 2006.

14. Titulaer MJ, Soffietti R, Dalmau J, Gilhus NE, Giometto B, Graus F, Grisold W, Honnorat J, Sillevis Smitt PA, Tanasescu R, et al: Screening for tumours in paraneoplastic syndromes: Report of an EFNS task force. Eur J Neurol 18: 19-e3, 2011. 